designing future studies to evaluate the use of dexamethasone in bacterial meningitis, were it not for the immense practical difficulties in recruitment for, and conduct of, such studies, which will be made immensely more difficult by the virtual disappearance of meningitis caused by Haemophilus influenzae type $b$ in developed countries.

I think that Prasad and Haines, while giving a complete picture of the characteristics of the perfect study, contribute very little to the readers' ability to use the available, if imperfect, data in the most clinically useful manner. The sensitivity analysis shown in table 3 is inappropriate and misleading, even as a worse case scenario. The number of subjects withdrawn from the analysis includes both children who are excluded because of diagnoses other than bacterial meningitis, primarily aseptic meningitis, and children with bacterial meningitis who were lost to follow up. It is clearly unreasonable to equally assign patients with aseptic meningitis to the treatment and control group and then assign all such patients in the treatment group as having had an adverse outcome and all patients in the control group as having had a favourable outcome as it is known that aseptic meningitis is rarely, if ever, associated with adverse sequelae. The only other work presented by the authors is a stratified analysis of the study by Girgis and coauthors which simply confirms that their overall findings of reduced mortality remained after stratification.

In summary, I think that only the most dramatic of treatment benefits in the most rigorous of trials could survive sensitivity analysis as exacting as that tabulated by Prasad and Haines. I do not consider that the results of this sensitivity analysis can reasonably be interpreted as showing lack of demonstrable benefit from dexamethasone in bacterial meningitis. In addition to the concern which the authors rightly have for demonstrating a favourable risk:benefit ratio in a real life clinical setting, there is also the concern that patients may be denied the benefit of an effective treatment which has been discounted by inappropriate and misleading handling of the data.

PETER MCINTYRE Westmead Hospital, Westmead NSW 2145, Australia

1 Prasad K, Haines T. Dexamethasone treatment for acute bacterial meningitis: how strong is the evidence for routine use? $f$ .Veurol Neurosurg Psychiatry 1995;59:31-7.

\section{Prasad replies:}

I read with interest the letter by McIntyre. He raises three points: (1) future studies will be difficult because of the virtual disappearance of meningitis in developed countries; (2) our sensitivity analysis is based on "unreasonable" assignment of adverse outcome to the treatment group, particularly for patients with aseptic meningitis and it cannot reasonably be interpreted as showing lack of demonstrable benefit from dexamethasone in bacterial meningitis; (3) the "patients may be denied the benefit of an effective treatment which has been discounted by inappropriate and misleading handling of the data". I take each point in turn.
If bacterial meningitis has virtually disappeared from the developed countries, future studies can and should be undertaken in developing countries where it is still a major public health problem. This is all the more necessary because maximum use of dexamethasone will occur where meningitis occurs. As we have shown in our paper, the studies to date have limited internal validity and generalisability.

Our sensitivity analysis is appropriate and revealing because it shows the weakness of the evidence available on the subject. This is what other authors have also recommended. ${ }^{1}$ McIntyre has an incorrect notion about the place and purpose of a sensitivity analysis. Firstly, this kind of analysis is required when authors do not report intention to treat (ITT) analysis or do not provide enough information to allow such an analysis and do not report steps taken to protect their analysis from bias. Most trials of treatments (whether dramatic or otherwise) do carry ITT analysis and therefore, sensitivity analysis is not required. If it is required, its purpose, as reported in our paper, is to assess the robustness of conclusions based on inadequate data and it is not to show the presence or lack of benefit. The inadequacy may be in terms of poor recording of risks or excessive losses to follow up. Almost any drug can be proved beneficial, if patients with adverse effects of the drug are withdrawn from the analysis or adverse effects are not counted. Although patients with aseptic meningitis rarely have a poor outcome, they are not immune to adverse effects of dexamethasone. Certainly, it is not reasonable to assume that all patients with aseptic meningitis given dexamethasone will have an adverse outcome, but to this extent, all sensitivity analyses with worst case scenarios are unreasonable. But it serves its purpose by showing that the published evidence is not strong and that it has not been proved beyond doubt that dexamethasone does more good than harm. However, "absence of proof" is not the "proof of absence". If we wish to base our practice on stronger evidence-as we should-we need more studies and proper analysis of the primary data collected from the investigators of the primary studies. Studies are proceeding in both directions. We and some investigators in Holland are conducting randomised trials of dexamethasone in adults with bacterial meningitis and a Cochrane review of the primary data is planned.

The third point by McIntyre is more profound. It has at its heart questions such as: "is it unethical to withhold a potentially effective treatment the risk-benefit profile of which is not studied adequately?"; "when are randomised trials of a treatment unethical?": "when is the evidence good enough to make strong recommendations?" and so on. These are issues about the science and ethics of practice of medicine. The available space will not allow me to do justice to the issues involved. I will simply state that until we have shown "a favourable risk-benefit ratio of a drug in real life clinical setting," there is no justification to subject all our patients to risk of adverse effects of the drug except in randomised trials. It is true that "ways of science" and evidence (randomised trials being the best method) do require denying some patients what may turn out to be beneficial, but the other way-policies advocating clinical use of a drug without proper evaluation of the risk-benefit ratiois far too dangerous to be acceptable.
1 Guyatt GH, Sackett DL, for the EvidenceBased Medicine Working Group. User guides to the medical literature, II: How to use an article about therapy or prevention, $\mathrm{A}$ Are the results of the study valid? $\Im A M A$ 1993;270:2598-601.

NOTICE

Announcement from the British Neuropsychiatry Association: 1996 summer meeting

The 1996 Summer meeting will be held on 14-16 July at Robinson College, Cambridge. It will include topics on neurodevelopment, language, and the presentation of short scientific papers and single case videos by members. The Association's AGM will be held on 16 July.

For further details of these meetings please contact: Sue Garratt, Administrative Assistant, BNPA, 17 Clocktower Mews, London N1 7BB. Telephone/Fax: 0171226 5949.

For details of membership of the BNPA, which is open to medical practitioners in psychiatry, neurology, and related clinical neurosciences, please contact: Dr fonathan Bird, Secretary BNPA, Burden Neurological Hospital, Stoke Lane, Stapleton, Bristol, BS16 1QT. Telephone: 01179701212 ext 2925/2929 or Sue Garratt at the address given above.

\section{BOOK REVIEWS}

All titles reviewed here are available from the BMJ Bookshop, PO Box 295, London WC1H 9TE. Prices include postage in the United Kingdom and for members of the British Forces Overseas, but overseas customers should add $£ 2$ per item for postage and packing. Payment can be made by cheque in sterling drawn on a United Kingdom bank, or by credit card (Mastercard, Visa or American Express) stating card number, expiry date, and your full name.

Atlas of Human Spinal Cord Evoked Potentials. Edited by MILAN R DIMITRIJEVIC and JOHN A HALTER. (Pp 180; £50.00). Published by Butterworth Heinemann, Oxford 1995. ISBN 0-75069631-1.

This is a multi-author book comprising a reference text for those readers with a highly 
specialised interest in the field of spinal cord evoked potentials (SCEPs). It reflects the growing body of knowledge on the electrophysiology of the spinal cord and the atlas itself presents a review of the nature, clinical features and intraoperative applications of SCEPs with a series of clear illustrations and examples of raw data, accompanied by a significant amount of explanatory text invoking almost 600 references. The reader is taken through the spinal cord physiology of evoked potentials (in contrast to the well established interest in associated cortical events). In addition to academic applications, the growth of intraoperative spinal monitoring means that this will become an important reference source for clinical neurophysiologists who are relatively new to this field or those who wish to optimise current techniques.

SIMON BONIFACE

Alternating Hemiplegia of Childhood. Edited by FREDERICK ANDERMANN, JEAN AICARDI and FEDERICO VIGEVANO. International Review of Child Neurology Series. Published by Raven Press.

This is an unusual book in which 50 authors contribute detailed accounts of a rare and tragic condition described in 75 individuals. Every aspect is fully covered including state of the art investigations and genetics but these have failed to reveal very much and the book remains essentially clinical. In the opening chapter $\mathrm{Dr}$ Steele (of SteeleRichardson fame) who first described alternating hemiplegia of childhood (AHC) exhorts that: "In this statistical era, it is imperative that individual observations and anecdotal accounts continue to be valued and encouraged". Such careful attention to detail has led the prodigious Professor Aicardi and others to tighten up the definition of AHC and reveal the existence of a most interesting phenomenon.

Affected children nearly always develop symptoms before the age of 18 months, and often in the neonatal period. Hemiplegia is not an early feature. Instead there are paroxysmal attacks apparently associated with distress, of abnormal limb or eye movements, dystonia and autonomic irregularities Developmental delay and static deficits such as ataxia and choreoathetosis develop later and there may be seizures. Attacks of flaccid hemiplegia which may occur on either side or bilaterally and are always abolished by sleep may not appear for a number of years. Flunarazine may attenuate the hemiplegias.

Little is known about the pathophysiology and the jury is still out on whether AHC is merely a severe form of hemiplegic migraine. Aicardi thinks not. The clinical features would suggest that the problem lies in the brain stem and may be bound up with sleep and other autonomic mechanisms. There are no pyramidal signs, but focal seizures might suggest that all is not well in the cortex. It has been suggested that mitochondrial function may be awry.

This scholarly book has not solved the mystery of AHC but its rigorous clinical definition provides science with its best chance in the future. It is well written and shows that the art of the clinical description remains useful and, as Steele advises, we should not abandon it.

REBECCA AYLWARD

Blue Books of Practical Neurology Movement Disorders 1 and 2 Reissue. Edited by $C$ D MARSDEN and $S$ FAHN. ( $P p$ 379 and $\mathrm{Pp} 468$ ). Published by ButterworthHeinemann Ltd, Oxford, 1995. ISBN 07506-2232-6.

There are few neurological books that are as influential as the Movement Disorder series from Butterworth-Heinemann. These books are not only beautifully written and crafted nto authorative texts but have over the years become the gold standard against which other books on movement disorders ar judged. It is therefore welcome to find that Butterworth-Heinemann have now reissued in a single volume Movement Disorders and 2 (MD1 and MD2) with no additions, deletions or amendments. These two books were originally published in 1982 and 1987 but have been out of print for some time, which has been especially frustrating to hose with an interest in movement disorders and possessing Movement Disorders 3 (MD3) published in 1994.

The decision to reissue MD1 and 2 is to be applauded not only because of the wealth of data it presents but also because it reminds neurologists how the field has evolved over the past 10 to 15 years. Thus although chapters in MD1 are now outdated, they nevertheless serve to illustrate the all too easily forgotten advances that have been made in this ever expanding field.

The new single volume book opens with an excellent preface from the editors, explaining the rationale for reissue of these books in an unmodified format (which even includes the odd typographical error!). The editors then go on in their preface to frame MD1 and 2 in a 1995 context, with the newer discoveries that the 1990s have brought. For example the discovery of multiple dopamine receptors and the revolutionary impact of molecular genetics. Although his helps in an understanding of the historical importance of the work presented in these books, it also highlights the redundancy of some chapters in this reissue such as the chapters on dopamine agonists in MD1 and molecular genetics in MD2.

Although critics may seize upon this point to claim that the book is redundant by virtue of its age, this is not the case. One of the strengths of these books is their attention to clinical detail and so many of the chapters, especially those in MD2, remain seminal in their presentation (for example, the chapters on PSP, Wilson's disease, and dystonia) Furthermore the fact that the books appeared at different times in the $1980 \mathrm{~s}$ highlights the advances that have taken place in the field of movement disorders, perhaps the best example being the use of botulinum toxin in focal dystonias and hemifacia spasm. MD1 discusses the various surgical approaches to dystonia in its penultimate chapter, which in MD2 is replaced by pharmacological approaches to the dystonic patient whilst in MD3 there is a whole chapter on the use of botulinum toxin. Of course it may be that in years to come the surgical approach to dystonia will re-surface in an analogous fashion to the surgical treatment of Parkinson's disease. Indeed changing fashions and trends in movement disorders are apparent in this reissue, with tardive dyskinesia being apparently of more interest in the 1980s. However, although some of these changes are related to the techniques of the day, in some cases it may reflect an embarrassing lack of progress in the understanding of certain disease states. One such example would seem to be Gilles de la Tourette's syndrome which is discussed fully in MD2, and about which little more is known eight years further on.

The question as to whom the reissued book will appeal is difficult, as older neurologists will have copies and those possessing MD3 may find inadequate reasons for purchasing MD1 and 2, especially as good review articles exist for most of the topics covered in this reissue. Indeed, although of historical interest, MD1 is largely out of date, especially when one takes into accoun the topics covered in MD3-for example, the cause, pathology, and nosology of parkinsonian syndromes. However, MD2 is less easy to dismiss on these grounds, and still represents an invaluable asset to the interested neurologist.

Yet despite these comments, I would not like to be without all three books, as they encapsulate in well written chapters the fascination and challenge of all aspects of movement disorders. Therefore no library should be without copies of all three books and all neurologists would benefit from reading them, not least as an illustration in the art of explaining on scientific and medical grounds complex neurological issues.

ROGER BARKER

Handbook of Dystonia. Edited by JOSEPH KING CHING TSUI and DONALD B CALNE. (Pp 504 \$165.00). Published by Marcel Dekker New York 1995. ISBN 0-8247-9515-6.

Dystonia is common and often treatable, two key elements that have really only emerged in the past decade. Hitherto, only three books specifically on dystonia have been published, all in the Advances in Neurology series, two of which (volumes 14 and 50) contained contributions from the first and second International Conferences on Dystonia in 1974 and 1986 (the third Conference will take place next year). The publication of this Handbook of Dystonia is therefore timely and welcome.

Almost all of the 39 contributors are from North America. The first six chapters deal with animal models, genetics, physiology, pathology and epidemiology of dystonia, and the next 10 on specific sites or causes of dystonia, the last of these contributions, by Barclay and Lang, giving an excellent overview of the complex subject of secondary dystonias. The last section deals first with medical and then surgical treatment, Bertrand and Lunz going into useful detail about their posterior primary ramicectomy operation for torticollis, before going on to deal with botulinum toxin in a further five chapters. Here we learn about its preparation (in minute detail probably of more interest to Saddam Hussein than to physicians), its properties and pharmacology, followed by good practical chapters 